ISSN 0103-9954

\title{
ASPECTOS FITOSSOCIOLÓGICOS DE FRAGMENTO DE FLORESTA ESTACIONAL DECIDUAL, SANTA MARIA, RS
}

\section{PHYTOSSOCIOLOGICAL ASPECTS OF FRAGMENT OF DECIDUAL SEASONAL FOREST, SANTA MARIA, RS}

\author{
Solon Jonas Longhi ${ }^{1}$ Maristela Machado Araujo ${ }^{2}$ Mônica Brucker Kelling ${ }^{3}$ \\ Juarez Martins Hoppe ${ }^{4}$ Ivanor Müller $^{5}$ Geedre Adriano Borsoi ${ }^{6}$
}

\section{RESUMO}

Foi realizado estudo da vegetação em fragmento de floresta estacional decidual em processo

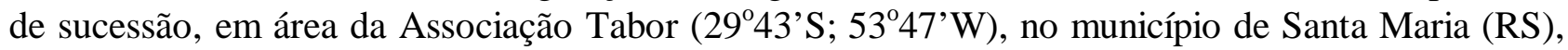
Brasil. Utilizaram-se dezesseis unidades amostrais de 10 × $20 \mathrm{~m}$ distribuídas de forma sistemática para avaliação (medição e identificação) dos indivíduos com Circunferência à Altura do Peito (CAP) igual ou maior de $30 \mathrm{~cm}$ e subunidades circulares com raios de $1,78 \mathrm{~m}$ para identificação e contagem da regeneração (indivíduos menores de $30 \mathrm{~cm}$ ). Observou-se a ocorrência de 64 espécies arbóreas e arbustivas de 54 gêneros e 31 famílias. As espécies com maior valor de importância foram Myrocarpus frondosus, Cupania vernalis, Ocotea puberula, Patagonula americana, Casearia sylvestris, Luehea divaricata, Enterolobium contortisiliquum e Helietta apiculata. Na regeneração, as espécies com maior densidade de indivíduos foram Trichilia elegans, Nectandra megapotamica, Actinostemon concolor, Piper gaudichaudianum, Cupania vernalis, Hybanthus bigibbosus, Aiouea saligna e Parapiptadenia rigida. Constatou-se o contínuo processo de sucessão por meio da substituição de espécies nos estratos da floresta e algumas bem-representadas desde a regeneração até o estrato superior.

Palavras-chave: fitossociologia, espécies florestais, sucessão, floresta estacional decidual.

\begin{abstract}
This work aimed at studying the vegetation in a fragment of Deciduous Seasonal Forest
\end{abstract}

1. Engenheiro Florestal, Dr., Professor do Departamento de Ciências Florestais, Centro de Ciências Rurais, Universidade Federal de Santa Maria, CEP 97105-900, Santa Maria (RS).

2. Engenheira Florestal, Doutoranda pelo Programa de Pós-Graduação em Engenharia Florestal, Centro de Ciências Rurais, Universidade Federal de Santa Maria, CEP 97105-900, Santa Maria (RS).

3. Engenheira Florestal, M.Sc., Professora do Colégio Agrícola de Santa Maria e Doutoranda pelo Programa de PósGraduação em Engenharia Florestal, Universidade Federal de Santa Maria, CEP 97105-900, Santa Maria (RS).

4. Engenheiro Florestal, M.Sc., Professor do Departamento de Ciências Florestais e Doutorando pelo Programa de Pós-Graduação em Engenharia Florestal, Centro de Ciências Rurais, Universidade Federal de Santa Maria, CEP 97105-900, Santa Maria (RS).

5. Engenheiro Florestal, M.Sc., Professor do Departamento de Estatística e Doutorando pelo Programa de PósGraduação em Engenharia Florestal, Centro de Ciências Rurais, Universidade Federal de Santa Maria, CEP 97105900, Santa Maria (RS).

6. Engenheiro Florestal, M.Sc., Acadêmico do Programa de Pós-Graduação em Engenharia Florestal, Centro de Ciências Rurais, Universidade Federal de Santa Maria, CEP 97105-900, Santa Maria (RS). 
under successional process, in an area of the Tabor Association $\left(29^{0} 43^{\prime} \mathrm{S} ; 53^{0} 47^{\prime} \mathrm{W}\right)$, municipal district of Santa Maria, RS, Brazil. Sixteen sample units $(10 x 20 \mathrm{~m})$ were systematically located in the area to allow the recording (measurement and indentification) of individual plants with a Circumference at Breast Height equal to or larger than $30 \mathrm{~cm}$. Sampling was also carried out in circular units with radii of $1,78 \mathrm{~m}$ to count up and identify the regeneration (individual with $\mathrm{CBH}$ smaller than $30 \mathrm{~cm}$ ). The occurrence of 64 species of trees and shrubs comprising 54 genera and 31 botanical families was observed. The most important species were Myrocarpus frondosus, Cupania vernalis, Ocotea puberula, Patagonula americana, Casearia sylvestris, Luehea divaricata, Enterolobium contortisiliquum and Helietta apiculata. In the regeneration the species with higher density of individuals were Trichilia elegans, Nectandra megapotamica, Actinostemon concolor, Piper gaudichaudianum, Cupania vernalis, Hybanthus bigibbosus, Aiouea saligna and Parapiptadenia rigida. It was demonstrated the evidence of a continuous successional process, through the replacement of species in the several strata of the forest, with some well represented from the regeneration up to the higher forest stratum.

Key words: phytossociology, forest species, succession, deciduous seasonal forest.

\section{INTRODUÇÃO}

No município de Santa Maria, ocorrem basicamente dois tipos de formações vegetais, os campos limpos e a floresta estacional decidual que cobre as escarpas da Serra Geral e vários morros testemunhos, situados na Depressão Central. Na região, uma grande proporção de floresta nativa sofreu processo de alteração antrópica, em circunstâncias do uso agrícola e desenvolvimento urbano, conseqüentemente, descaracterizando a estrutura natural. Atualmente, existem fragmentos da floresta com vegetação nativa em vários estágios de sucessão.

Para a caracterização da vegetação arbórea de uma determinada área, é necessário reconhecer as espécies presentes no local e fazer uma avaliação da estrutura horizontal e vertical da floresta, com o objetivo de verificar seu desenvolvimento. A estrutura horizontal permite a determinação da densidade, dominância, freqüência e importância das espécies na floresta e a estrutura vertical analisa o estágio de desenvolvimento desta floresta, com base na distribuição das espécies nos diferentes estratos. A obtenção de dados para determinação desses parâmetros, segundo BEARZI et al. (1992), permite o estudo de regeneração e recuperação de uma área florestal, bem como sobre a evolução da floresta ao longo do tempo, próximo da área de estudo.

Estudos sobre a estrutura fitossociológica da floresta estacional decidual da região de Santa Maria (RS) já foram efetuados por SILVA \& LONGHI (1989), MACHADO \& LONGHI (1992), BEARZI et al. (1992), DIAS et al. (1996) e LONGHI et al. (1999), em diferentes locais do município, sendo que o trabalho realizado por DIAS et al. (1996) foi efetuado em dois morros testemunha, o Cerrito e o Cerro Mariano.

O presente estudo visou à caracterização da floresta da Associação Tabor, importante recurso natural de Santa Maria (RS) que, atualmente, se encontra em regime de preservação permanente.

Ciência Florestal, v. 10 , n. 2, 2000 


\section{REVISÃO DE LITERATURA}

A fitossociologia estuda o agrupamento das plantas, sua interrelação e dependência aos fatores bióticos em determinado ambiente, ou seja, cada indivíduo que habita determinado local atua sobre os demais, assim como os fatores externos (BRAUN-BLANQUET, 1979).

Estudos fitossociológicos podem ser realizados mediante análise da estrutura horizontal da floresta, obtida por parâmetros como densidade, freqüência e dominância das espécies (CAIN \& CASTRO, 1959) e estrutura vertical (posição sociológica e regeneração) (FINOL, 1971). Esses parâmetros caracterizam a condição de ocorrência em que se encontram as espécies e, quando somados na forma relativa, definem o VI (valor de importância) de uma espécie em relação às demais existentes na floresta.

O conceito e utilização desses parâmetros está vastamente explorada na literatura, podendose citar alguns trabalhos como o de LAMPRECHT (1964), FINOL (1975), LONGHI (1980), BARROS (1986), MARTINS (1991) e VIEIRA (1996) que descrevem a densidade absoluta (DA) como o número total de indivíduos de uma espécie em determinada área e a densidade relativa (DR) como a participação de uma determinada espécie sobre as demais. A frequiência absoluta (FA) mostra a ocorrência de cada espécie no total de unidades amostradas e a freqüência relativa (FR) expressa a frequiência de uma espécie em relação às outras, sendo um parâmetro utilizado para dar uma visão de como as espécies se distribuem na área, enquanto a dominância absoluta (DoA) é a forma de expressar o espaço de superfície horizontal ocupado por determinada espécie, sendo calculada por meio da área basal, e a Dominância relativa (DoR) expressa o espaço horizontal que uma espécie está ocupando, em relação às outras .

A inferência sobre a hierarquia das espécies por meio do valor de importância pode ser realizado de forma mais precisa, quando associado a cada um dos parâmetros, pois algumas espécies têm a importância aumentada em razão, apenas, de um parâmetro (ARAUJO, 1998). LONGHI (1997) também hierarquizou as espécies da floresta de acordo com o valor de cobertura (VC), o qual consiste em considerar apenas o número de árvores e suas dimensões. Esse valor é obtido através da soma da densidade e dominância relativa da espécies, calculando o espaço que ocupa e desconsiderando a forma em que a espécie se distribui.

A regeneração natural é o estágio que segue a independência da plântula da reserva da semente e precede o estado vegetativo adulto e o reprodutivo, ou seja, é o indivíduo jovem na floresta. Esse estágio é de grande importância, já que o fracasso dos processos adaptativos, nesse período, pode eliminar a espécie do local (AMO RODRÍGUEZ \& GÓMEZ-POMPA, 1976). O estudo da regeneração natural de florestas tropicais e subtropicais é de grande importância para a recuperação de ecossistemas que sofrem alterações, permitindo o conhecimento inicial da sucessão secundária (GÓMES-POMPA \& WIECHERS, 1976).

A regeneração pode ser avaliada por meio da freqüência, densidade e categorias de tamanho (indivíduos são avaliados por classes de tamanho), sendo considerado que, quanto maior for o indivíduo, maior também será sua possibilidade de permanecer na área (FINOL, 1971; JARDIM \& HOSOKAWA, 1986). Outros trabalhos têm a regeneração avaliada apenas pela densidade de 
indivíduos por espécie (LONGHI et al.; 1999; VIEIRA, 1996). A regeneração associada à posição sociológica foi utilizada por FINOL (1971) e ARAUJO (1998) para descrever a estrutura vertical, enquanto LONGHI et al. (1999) utilizam o perfil longitudinal para realizar tal avaliação.

A diversidade da vegetação pode ser observada por vários índices, sendo que o de Shannon é o mais utilizado para expressar as características da comunidade, pelo seu nível de organização biológica (BROWER \& ZAR, 1984). Esse índice leva em consideração o número de espécies diferentes no povoamento e a proporção de cada espécie (PIELOU, 1977).

\section{MATERIAL E MÉTODOS}

\section{Descrição da Área}

A área de estudo situa-se no município de Santa Maria (RS) nas cordenadas $29^{\circ} 43^{\prime}$ de latitude sul e $53^{\circ} 47^{\prime}$ de longitude oeste, com altitude máxima de $245 \mathrm{~m}$, sendo propriedade da Associação Tabor. Sua vegetação é caracterizada por Floresta Estacional Decidual. O fragmento de floresta estudado é resultado da regeneração natural, após muito tempo de exploração, encontrandose em fase adiantada de sucessão, atualmente em regime de preservação permanente.

A região pertence à Bacia do Paraná, com Cobertura Sedimentar Gonduânica, pertencente à Formação Botucatu, constituída por arenito de granulação fina a média, sendo produto de vulcanismo ácido e básico (KAUL, 1990). A unidade de relevo pertence à Depressão Periférica da Bacia do Paraná, que entrepõe-se entre rochas efusivas e cristalinas constituídas pelas formações sedimentares Gonduânicas e modeladas pelos processos erosivos, não apresentando continuidade espacial e caracterizando relevo suave ondulado a ondulado (HERRMANN \& ROSA, 1990).

Segundo SARTORI (1979), a região de Santa Maria está situada praticamente na zona de transição entre a Depressão Periférica Sul-Rio-Grandense, também conhecida por Depressão Central e a escarpa arenito-basáltica do Planalto Meridional Brasileiro. A leste, sudeste e nordeste da cidade, em meio à área sedimentar, destacam-se vários morros testemunhas da Serra Geral, capeados por rochas basálticas e granófiros que atuaram como camadas mantenedoras da topografia, como o Cerro Mariano da Rocha, onde se localiza a área estudada. Esse morro situa-se à cerca de $1.500 \mathrm{~m}$ da escarpa da Serra Geral. Os morros testemunhas são constituídos geologicamente por arenitos da Formação Botucatu (eólicos e fluviais) na base, seguindo-se um derrame de basalto e arenito intercalar da Formação Serra Geral, rumo ao topo. Nesses morros, a declividade situa-se entre 16,6 a $21 \%$.

De acordo com as características do local, o solo pertence à Unidade de Mapeamento Charrua, solos litólicos eutróficos, textura média, relevo montanhoso e substrato basalto, sendo solos pouco desenvolvidos, rasos e moderadamente drenados. Nessa unidade taxonômica, observamse em geral afloramentos de rochas (BRASIL, 1973).

O clima da região, conforme classificação de Köppen, é do tipo Cfa, subtropical de clima temperado chuvoso cuja temperatura média do mês mais quente é $24,8^{\circ} \mathrm{C}$ em janeiro e a temperatura média do mês mais frio, em julho, é $14,1^{\circ} \mathrm{C}$. A precipitação média anual é de 1.769 
mm/ano (MORENO, 1961).

\section{Metodologia}

Foram levantadas dezesseis unidades amostrais de 10 x $20 \mathrm{~m}\left(200 \mathrm{~m}^{2}\right)$, distribuídas de forma sistemática e mantendo uma distância de $30 \mathrm{~m}$ entre amostras que seguiam a mesma orientação da declividade. Consideraram-se, em cada unidade amostral, todas as espécies arbóreas e arbustivas com CAP (circunferência à altura do peito) maior ou igual a $30 \mathrm{~cm}$, para as quais foram registrados os seguintes dados: espécie vegetal, CAP, altura total, altura comercial, qualidade do fuste e a localização dos indivíduos na parcela (coordenadas das árvores).

Além desses dados, foi registrada a posição sociológica de cada árvore, considerando três estratos: inferior (3); médio (2); e superior (1). Os estratos foram avaliados visualmente por um membro da equipe, apresentando amplitude variável que dependia da altura máxima dos indivíduos presentes na parcela e na sua volta. Assim, as espécies puderam ser consideradas dentro do estrato em que ocorriam. A estrutura vertical da floresta foi visualizada, mais detalhadamente, através do por meio longitudinal de uma das amostras escolhidas aleatoriamente.

Altura total, altura comercial, localização do indivíduo na parcela e qualidade do fuste foram utilizados na esquematização do perfil vertical da floresta, permitindo mais informações do ponto em que ocorreu o indivíduo, a qualidade e inserção dos galhos no fuste.

Para avaliar a regeneração, foram alocadas, no final de cada parcela, subunidades amostrais circulares com raio de $1,78 \mathrm{~m}\left(10 \mathrm{~m}^{2}\right)$. Nessas unidades, foram contados todos os indivíduos arbóreos e arbustivos, por espécie, com altura maior de $10 \mathrm{~cm}$ e CAP menor de $30 \mathrm{~cm}$.

Otimizou-se a intensidade amostral por meio da curva espécie/área, a qual avalia a amostragem mínima a ser realizada em uma comunidade vegetal (JARDIM \& HOSOKAWA, 1986; ARAUJO, 1998; LONGHI et al., 1999)

A identificação das espécies foi realizada a campo, sendo que os indivíduos não identificados, no local, tiveram material botânico coletado para posterior identificação no Herbário do Departamento de Ciências Florestais da Universidade Federal de Santa Maria.

Para análise da estrutura horizontal da vegetação, utilizou-se o programa FITOPAC 1 (desenvolvido pelo professor G. J. Shepherd, do Departamento de Botânica da UNICAMP, em 1988), em que se obteve valores de densidade, freqüência e dominância absolutas e relativas; valores de importância e de cobertura das espécies, além do índice de diversidade de Shannon.

\section{RESULTADOS E DISCUSSÃO}

\section{Relação número de espécies - área}

Verificou-se que as parcelas amostrais levantadas foram suficientes para representar a composição florística da área estudada, sendo possível observar tendência à estabilização na curva espécie-área (Figura 1). Ocorreu uma estabilização parcial, entre 1.400 e $2.100 \mathrm{~m}^{2}$ da área amostral. 
No entanto, a medida que foi aumentando a altitude, houve um acréscimo no número de espécies, voltando a se estabilizar em torno dos $2.400 \mathrm{~m}^{2}$, apesar de, na última parcela, ocorrer o acréscimo de uma nova espécie, havendo uma pequena desestabilização da curva.

O fato de não ocorrer uma estabilização nítida é em consequiência da característica heterogênea da floresta que, a cada parcela locada, apresentou uma nova espécie, porém a tendência à estabilização, na parcela, pode ser considerada suficiente para indicar o número de parcelas a ser utilizado (JARDIM \& HOSOKAWA, 1986), ou seja, as dezesseis parcelas foram suficientes para representar a vegetação na área.

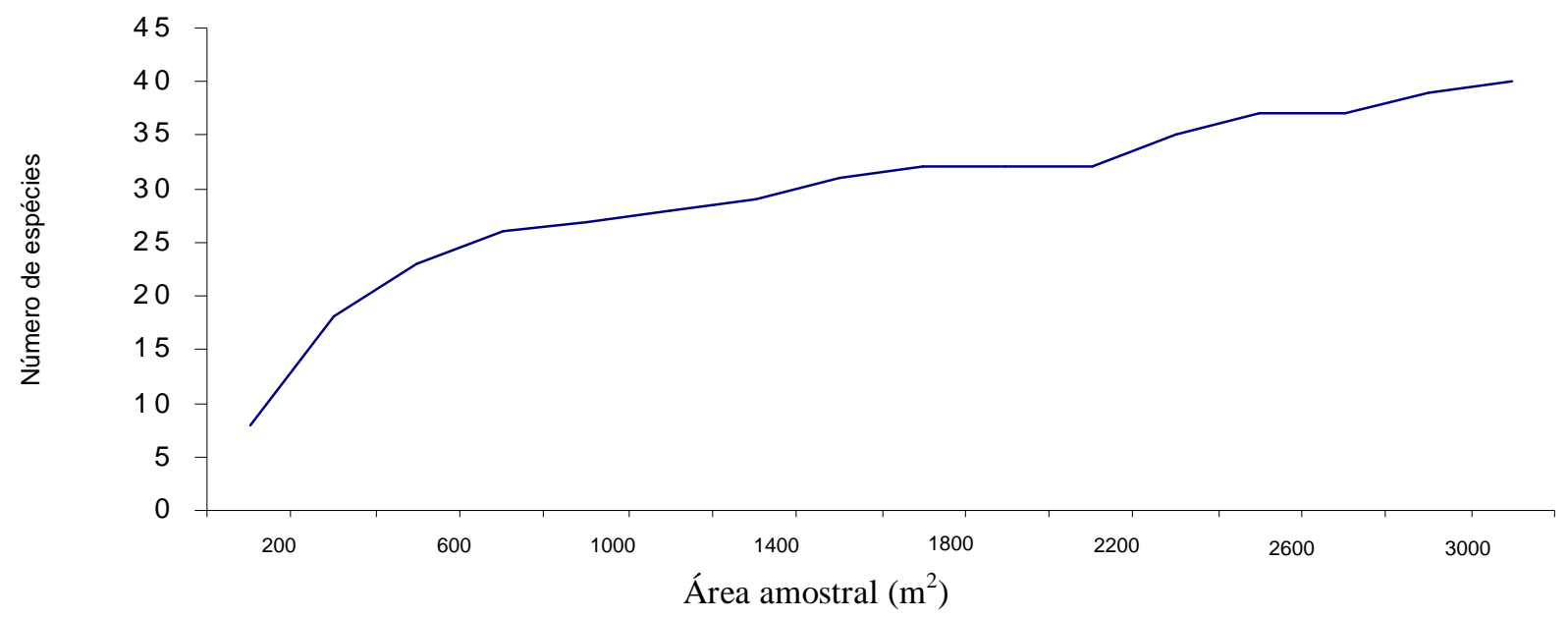

FIGURA 1: Curva espécie-área da Floresta Estacional Decidual estudada em Santa Maria (RS).

\section{Composição florística}

$\mathrm{Na}$ área estudada foram amostradas 64 espécies arbóreas e arbustivas, constituindo os diferentes estratos da floresta, pertencentes a 52 gêneros, distribuídos em 31 famílias botânicas (Tabela 1). Essa riqueza florística foi semelhante àquela encontrada por DIAS et al. (1996), em dois morros testemunha na mesma região onde foram observadas 70 espécies de 58 gêneros e 29 famílias.

Nos estratos superiores (indivíduos com CAP maior ou igual a $30 \mathrm{~cm}$ ) foram identificadas quarenta espécies arbóreas, enquanto que as 23 espécies restantes somente ocorreram na regeneração natural.

Os gêneros que apresentaram maior número de espécies foram Eugenia (3), Trichilia (3), Sebastiania (2), Chrysophyllum (2), Allophylus (2), Myrsine (2), Nectandra (2), Ocotea (2) e as famílias que mais se destacaram foram Lauraceae, Meliaceae e Mimosaceae, representadas por cinco espécies, e as famílias Myrtaceae e Sapindaceae, com quatro espécies cada.

A floresta apresentou elevada diversidade onde se constatou que o Índice de Shannon foi igual a 3,213, indicando uma distribuição mais uniforme do número de indivíduos em relação ao número de espécies, quando comparado com LONGHI et al. (1999), em estudo realizado em outro fragmento florestal da região, identificando maior riqueza florística (79 espécies), porém menor 
índice de diversidade $(3,12)$.

TABELA 1: Espécies, família e nome comum de árvores e arbustos amostrados em um fragmento florestal na Depressão Central, Santa Maria (RS).

\begin{tabular}{|c|c|c|}
\hline Família & Espécie & Nome comum \\
\hline$\overline{\text { Annonaceae }}$ & Rollinia rugulosa Schlechtendal & Araticum \\
\hline Arecaceae & Syagrus romanzoffiana (Cham.) Glass. & Jerivá \\
\hline Asteraceae & Dasyphyllum spinescens (Less.) Cabr. & Açucará \\
\hline Bignoniaceae & Tabebuia impetiginosa (Mart.) Sandw. & Ipê-roxo \\
\hline Bombacaceae & Chorisia speciosa St. Hil. & Paineira \\
\hline Bombacaceae & Xylosma pseudosalzmannii Sleumer & Espinho-judeu \\
\hline Boraginaceae & Cordia trichotoma (Vell.) Arrab. ex Steud. & Louro \\
\hline Boraginaceae & Patagonula americana $\mathrm{L}$. & Guajuvira \\
\hline Ebenaceae & Diospyros inconstans Jacq. & Maria-preta \\
\hline Euphorbiaceae & Actinostemon concolor (Spreng.) Müll. Arg. & Laranjeira-do-mato \\
\hline Euphorbiaceae & Sebastiania brasiliensis Spreng. & Branquilho-leiteiro \\
\hline Euphorbiaceae & Sebastiania commersoniana (Baill.) L. B. Smith \& R. J. Downs & Branquilho-leiteiro \\
\hline Fabaceae & Apuleia leiocarpa (Vog.) Macbr. & Grápia \\
\hline Fabaceae & Machaerium stipitatum Vogel & Canela-do-brejo \\
\hline Fabaceae & Myrocarpus frondosus Allem. & Cabriúva \\
\hline Flacourtiaceae & Banara tomentosa Clos & Guaçatunga-branca \\
\hline Flacourtiaceae & Casearia sylvestris $\mathrm{Sw}$. & Chá-de-bugre \\
\hline Lauraceae & Aiouea saligna Meissner & Canela-vermelha \\
\hline Lauraceae & Nectandra lanceolata Nees et Mart. ex Nees & Canela-amarela \\
\hline Lauraceae & Nectandra megapotamica (Spreng.) Mez & Canela-preta \\
\hline Lauraceae & Ocotea puberula Nees & Canela-guaicá \\
\hline Lauraceae & Ocotea sp. & Canela \\
\hline Meliaceae & Cabralea canjerana (Vell.) Mart. & Canjerana \\
\hline Meliaceae & Cedrela fissilis Vell. & Cedro \\
\hline Meliaceae & Trichilia catigua A. Juss. & Catiguá-verdadeiro \\
\hline Meliaceae & Trichilia claussenii C. DC. & Catiguá-vermelho \\
\hline Meliaceae & Trichillia elegans A. Juss. & Pau-de-ervilha \\
\hline Mimosaceae & Acacia bonariensis Gill. ex Hook. et Arn. & Unha-de-gato \\
\hline Mimosaceae & Calliandra tweediei Benth. & Angiquinho \\
\hline Mimosaceae & Enterolobium contortisiliquum (Vell.) Morong. & Timbaúva \\
\hline Mimosaceae & Inga marginata Willd. & Ingá-feijão \\
\hline Mimosaceae & Parapiptadenia rigida (Benth.) Brenan & Angico-vermelho \\
\hline Moraceae & Ficus luschnathiana Miq. & Figueira-do-mato \\
\hline Moraceae & Maclura tinctoria (L.) Don ex Stendel & Tajuba \\
\hline Moraceae & Sorocea bonplandii (Baill.) Burger, Lanjow \& Boer & Cincho \\
\hline Myrsinaceae & Myrsine lorentziana $\mathrm{Mez}$ & Capororoca \\
\hline Myrsinaceae & Myrsine umbellata Mart. & Capororocão \\
\hline Myrtaceae & Eugenia involucrata D.C. & Cerejeira \\
\hline Myrtaceae & Eugenia rostrifolia Legr. & Batinga \\
\hline
\end{tabular}


TABELA 1: Continuação ...

\begin{tabular}{|c|c|c|}
\hline Família & Espécie & Nome comum \\
\hline Myrtaceae & Eugenia uniflora $\mathrm{L}$. & Pitangueira \\
\hline Myrtaceae & Myrcianthes pungens (Berg) Legr. & Guabiju \\
\hline Phytolaccaceae & Phytolacca dioica $\mathrm{L}$. & Umbu \\
\hline Piperaceae & Piper gaudichaudianum Kunth & Pimentinha \\
\hline Polygonaceae & Ruprechtia laxiflora Meiss. & Marmeleiro-do-mato \\
\hline Rosaceae & Prunus sellowii Koehne & Pessegueiro-do-mato \\
\hline Rubiaceae & Faramea marginata Cham. & Pimenteira \\
\hline Rutaceae & Helietta apiculata Benth. & Canela-de-veado \\
\hline Rutaceae & Zanthoxylum rhoifolium $\mathrm{L}$. & Mamica-de-cadela \\
\hline Sapindaceae & Allophylus edulis (St. Hil.) Radlk. & Chal-chal \\
\hline Sapindaceae & Allophylus guaraniticus Camb. & Vacum \\
\hline Sapindaceae & Cupania vernalis Camb. & Camboatá-vermelho \\
\hline Sapindaceae & Matayba elaeagnoides Radlk. & Camboatá-branco \\
\hline Sapotaceae & Sideroxylon obtusifolium (Roem. \& Schult.) Penning & Sombra-de-touro \\
\hline Sapotaceae & Chrysophyllum gonocarpum (Mart. \& Eich.) Engl. & Aguaí-da-serra \\
\hline Sapotaceae & Chrysophyllum marginatum (Hook. et Arn.) Radlk. & Aguaí-leiteiro \\
\hline Solanaceae & Não identificada & \\
\hline Solanaceae & Solanum pseudoquina St. Hil. & Peloteiro \\
\hline Styracaceae & Styrax leprosum Hook. et Arn. & Carne-de-vaca \\
\hline Thymeliaceae & Daphnopsis racemosa Griseb. & Imbira \\
\hline Tiliaceae & Luehea divaricata Mart. & Açoita-cavalo \\
\hline Ulmaceae & Celtis spinosa Spreng. & Taleira \\
\hline Urticaceae & Urera baccifera Gaud. & Urtigão-do-mato \\
\hline Violaceae & Hybanthus bigibbosus (St. Hil.) Hassl. & Viuvinha \\
\hline- & Não identificada & - \\
\hline
\end{tabular}

\section{Estrutura da vegetação}

A análise da estrutura horizontal da floresta permitiu que se constatasse que as espécies com maior valor de importância são Myrocarpus frondosus (33,58), Cupania vernalis $(26,89)$, Ocotea puberula (16,51), Patagonula americana (16,40), Casearia sylvestris $(15,48)$, Luehea divaricata $(15,42)$, Enterolobium contortisiliquum $(14,76)$ e Helietta apiculata $(13,01)$, conforme pode ser observado na Tabela 2. Entre essas espécies, Ocotea puberula e Enterolobium contortisiliquum tiveram sua importância aumentada, principalmente, por causa da dominância relativa, ou seja, apresentaram indivíduos com maior diâmetro o que, conseqüentemente, influi no aumento da área basal da espécie e no parâmetro dominância.

Em contrapartida, no caso de Casearia sylvestris, a maior importância foi definida, principalmente, em razão do número de indivíduos (densidade) e de sua distribuição na área (freqüência). Conseqüientemente, houve a diminuição no valor de cobertura dessa espécie $(9,51)$, pois, apesar do grande número de indivíduos, estes se apresentam com pequenos diâmetros. Nas demais espécies citadas, foi observado que todos os parâmetros contribuíram de forma relativamente uniforme para a determinação do valor de importância. 
TABELA 2: Parâmetros fitossociológicos das espécies amostradas, com CAP $\geq 30 \mathrm{~cm}$ em um fragmento florestal na Depressão Central, Santa Maria (RS).

\begin{tabular}{|c|c|c|c|c|c|c|c|c|c|}
\hline Espécie & $\mathrm{N}$ & DA & FA & DoA & DR & FR & DoR & VI & $\mathrm{VC}$ \\
\hline Mvrocarpus frondosus & 31 & 96.9 & 62.50 & 7.4129 & 11.36 & 7.46 & 14.77 & 33.58 & 26.12 \\
\hline Cupania vernalis & 34 & 106,3 & 50,00 & 4,2505 & 12,45 & 5.97 & 8,47 & 26,89 & 20,92 \\
\hline Ocotea puberula & 13 & 40,6 & 18,75 & 4,7716 & 4,76 & 2,24 & 9.50 & 16,51 & 14,27 \\
\hline Patagonula americana & 19 & 59.4 & 43,75 & 2,1156 & 6.96 & 5,22 & 4,21 & 16,40 & 11.17 \\
\hline Casearia svlvestris & 20 & 62.5 & 50,00 & 1,0944 & 7.33 & 5.97 & 2,18 & 15,48 & 9.51 \\
\hline Luehea divaricata & 11 & 34.4 & 43,75 & 3,0956 & 4,03 & 5,22 & 6,17 & 15,42 & 10,20 \\
\hline Enterolobium contortisiliquиm & 8 & 25,0 & 31,25 & 4,0673 & 2.93 & 3,73 & 8,10 & 14,76 & 11,03 \\
\hline Helietta apiculata & 15 & 46,9 & 31.25 & 1,8997 & 5,49 & 3.73 & 3,78 & 13,01 & 9.28 \\
\hline Morta & 10 & 31.3 & 50,00 & 0,9432 & 3,66 & 5.97 & 1,88 & 11.51 & 5.54 \\
\hline Chrysophyllum marginatum & 9 & 28,1 & 25,00 & 1,9676 & 3,30 & 2.99 & 3,92 & 10,20 & 7.22 \\
\hline Rollinia rugulosa & 8 & 25,0 & 31,25 & 1,0378 & 2.93 & 3,73 & 2.07 & 8,73 & 5,00 \\
\hline Trichilia claussenii & 10 & 31,3 & 31,25 & 0,6322 & 3,66 & 3,73 & 1.26 & 8,65 & 4,92 \\
\hline Actinostemon concolor & 10 & 31,3 & 31,25 & 0,3268 & 3,66 & 3,73 & 0,65 & 8,05 & 4,31 \\
\hline Nectandra megapotamica & 7 & 21.9 & 18,75 & 1,2174 & 2.56 & 2,24 & 2,42 & 7,23 & 4,99 \\
\hline Nectandra lanceolata & 6 & 18.8 & 18,75 & 1,1386 & 2.20 & 2,24 & 2.27 & 6.70 & 4,47 \\
\hline Phvtolacca dioica & 5 & 15,6 & 12,50 & 1.5889 & 1,83 & 1.49 & 3,16 & 6,49 & 5,00 \\
\hline Parapiptadenia rigida & 4 & 12.5 & 25.00 & 0,801 & 1.47 & 2.99 & 1.60 & 6,05 & 3,06 \\
\hline Ficus luschnathiana & 2 & 6,3 & 12,50 & 1,8788 & 0,73 & 1,49 & 3,74 & 5,97 & 4,48 \\
\hline Chorisia speciosa & 1 & 3,1 & 6.25 & 2,3591 & 0,37 & 0,75 & 4.70 & 5,81 & 5,07 \\
\hline Prunus sellowii & 5 & 15,6 & 25,00 & 0,399 & 1.83 & 2,99 & 0,80 & 5.61 & 2.63 \\
\hline Sorocea bonplandii & 4 & 12.5 & 25,00 & 0,142 & 1,47 & 2.99 & 0,28 & 4,73 & 1.75 \\
\hline Chrysophyllum gonocarpum & 5 & 15.6 & 18,75 & 0,307 & 1,83 & 2,24 & 0,61 & 4,68 & 2,44 \\
\hline Sideroxvlon obtusifolium & 3 & 9.4 & 18,75 & 0.641 & 1,10 & 2,24 & 1.28 & 4,61 & 2.38 \\
\hline Eugenia rostrifolia & 5 & 15.6 & 12.50 & 0.624 & 1,83 & 1,49 & 1.24 & 4,57 & 3,07 \\
\hline Ruprechtia laxiflora & 3 & 9,4 & 12,50 & 0,952 & 1,10 & 1,49 & 1,90 & 4,49 & 3,00 \\
\hline Tabebuia impetiginosa & 2 & 6.3 & 12.50 & 1,0091 & 0,73 & 1,49 & 2.01 & 4,24 & 2,74 \\
\hline Maclura tinctoria & 3 & 9.4 & 18,75 & 0,403 & 1,10 & 2,24 & 0,80 & 4,14 & 1,90 \\
\hline Mvrcianthes pungens & 3 & 9,4 & 12,50 & 0.693 & 1,10 & 1,49 & 1,38 & 3,97 & 2,48 \\
\hline Mvrsine lorentziana & 2 & 6,3 & 12.50 & 0,352 & 0.73 & 1,49 & 0,70 & 2.93 & 1.43 \\
\hline Apuleia leiocarpa & 1 & 3,1 & 6,25 & 0,653 & 0,37 & 0,75 & 1.30 & 2,41 & 1,67 \\
\hline Stvrax leprosum & 3 & 9.4 & 6.25 & 0,232 & 1,10 & 0,75 & 0,46 & 2.31 & 1.56 \\
\hline Machaerium stipitatum & 1 & 3,1 & 6.25 & 0.407 & 0.37 & 0,75 & 0,81 & 1,92 & 1,18 \\
\hline Zanthoxvlum rhoifolium & 1 & 3,1 & 6.25 & 0.285 & 0.37 & 0,75 & 0.57 & 1.68 & 0,93 \\
\hline Sebastiania commersoniana & 2 & 6.3 & 6.25 & 0,085 & 0,73 & 0.75 & 0,17 & 1,65 & 0,90 \\
\hline Aiouea saligna Meissner & 1 & 3,1 & 6.25 & 0,133 & 0,37 & 0,75 & 0.26 & 1,38 & 0,63 \\
\hline Cabralea canierana & 1 & 3,1 & 6.25 & 0,115 & 0,37 & 0.75 & 0,23 & 1,34 & 0,60 \\
\hline Mvrsine umbellata & 1 & 3,1 & 6.25 & 0,055 & 0,37 & 0,75 & 0,11 & 1,22 & 0,48 \\
\hline Banara tomentosa & 1 & 3,1 & 6.25 & 0,042 & 0.37 & 0,75 & 0.08 & 1.20 & 0,45 \\
\hline Solanum pseudoquina & 1 & 3,1 & 6.25 & 0,027 & 0,37 & 0,75 & 0.05 & 1,17 & 0.42 \\
\hline Trichillia elegans & 1 & 3,1 & 6.25 & 0,024 & 0,37 & 0,75 & 0.05 & 1,16 & 0,41 \\
\hline Allophvlus edulis & 1 & 3,1 & 6,25 & 0,024 & 0,37 & 0.75 & 0.05 & 1,16 & 0,41 \\
\hline Total & 273 & 853.4 & 837.5 & 50.2031 & 100 & 100 & 100 & 300 & 200 \\
\hline
\end{tabular}

Em que: $\mathrm{N}$ = número de indivíduos; $\mathrm{DA}=$ densidade absoluta; FA = freqüência absoluta; DoA = dominância absoluta; $\mathrm{DR}=$ densidade relativa; $\mathrm{FR}=$ frequiência relativa; $\mathrm{DoR}=$ dominância relativa; $\mathrm{VI}=$ valor de importância; $\mathrm{VC}=$ valor de cobertura.

Quando as espécies foram hierarquizadas pelo valor de cobertura, houve a seguinte seqüencia: Myrocarpus frondosus $(26,12)$, Cupania vernalis $(20,92)$, Ocotea puberula $(14,27)$, Patagonula americana (11,17), Enterolobium contortisiliquum (11,03), Luehea divaricata (10,20), Casearia sylvestris $(9,51)$ e Helietta apiculata $(9,28)$. Apesar da modificação na posição hierárquica das últimas quatro espécies, quando se avalia pelo valor de cobertura, observa-se que estas permanecem sendo as oito espécies mais representativas na vegetação, o que as confirma como principais componentes, indiferente ao "índice" utilizado para avaliação. 
Foi observado um grande número de indivíduos mortos na área $(3,66 \%)$, provavelmente em conseqüência do estágio sucessional em que a floresta se encontra, fato este já observado por DIAS et al. (1996) em levantamento realizado na mesma região onde observaram a ocorrência de 4,78\% de indivíduos mortos, evidenciando a grande concorrência pela luz das espécies componentes da floresta.

$\mathrm{Na}$ análise da estrutura vertical (Tabela 3), verificou-se que no estrato superior (Estrato 1)

TABELA 3: Valor de importância (VI) por estrato de árvores e arbustos, com CAP $\geq 30 \mathrm{~cm}$ amostrados em um fragmento florestal na Depressão Central, Santa Maria (RS).

\begin{tabular}{|c|c|c|c|}
\hline Espécie & VI (Est 1) & VI (Est 2) & VI (Est 3) \\
\hline $\begin{array}{l}\text { Actinostemon concolor } \\
\text { Aiouea saliong }\end{array}$ & & & 19.97 \\
\hline Aiouea saligna & 3,84 & & \\
\hline Allophylus edulis & & & 2,50 \\
\hline Apuleia leiocarpa & 5,45 & & \\
\hline Banara tomentosa & & & 1,50 \\
\hline Sideroxylon obtusifolium & & 7,05 & 7,44 \\
\hline Cabralea canjerana & 3,79 & & \\
\hline Casearia sylvestris & & 6,88 & 38,39 \\
\hline Chorisia speciosa & 10,73 & & \\
\hline Chrysophyllum gonocarpum & & 8,05 & 7,16 \\
\hline Chrysophyllum marginatum & 11,02 & 13,02 & 7,69 \\
\hline Cupania vernalis & 12,54 & 42,28 & 41,13 \\
\hline Enterolobium contortisiliquum & 31,69 & 5,41 & \\
\hline Ficus luschnathiana & 9,08 & 3,72 & \\
\hline Helietta apiculata & 16,40 & 24,06 & 2,55 \\
\hline Luehea divaricata & 21,29 & 21,00 & 6,01 \\
\hline Machaerium stipitatum & 4,69 & & \\
\hline Maclura tinctoria & 4,40 & 3,84 & 2,50 \\
\hline Myrcianthes pungens & & 11,95 & 3,15 \\
\hline Myrocarpus frondosus & 56,16 & 28,08 & 15,28 \\
\hline Eugenia rostrifolia & 8,35 & 4,06 & 5,05 \\
\hline Myrsine umbellata & & 3,74 & \\
\hline Nectandra lanceolata & 10,94 & 8,23 & 3,11 \\
\hline Myrsine lorentziana & & 6,05 & 2,57 \\
\hline Nectandra megapotamica & 4,43 & 19,28 & 3,50 \\
\hline Ocotea puberula & 28,52 & 16,21 & \\
\hline Parapiptadenia rigida & 9,05 & 3,91 & 2,47 \\
\hline Patagonula americana & 8,89 & 19,60 & 27,54 \\
\hline Phytolacca dioica & 14,23 & & 3,45 \\
\hline Prunus sellowii & & 4,44 & 12,68 \\
\hline Rollinia rugulosa & & 22,19 & 8,35 \\
\hline Ruprechtia laxiflora. & 6,10 & & 4,42 \\
\hline Sebastiania commersoniana & & & 4,30 \\
\hline Solanum pseudoquina & & & 2,55 \\
\hline Sorocea bonplandii & & & 10,73 \\
\hline Styrax leprosum & & 6,00 & 3,11 \\
\hline Tabebuia impetiginosa & 9,99 & & \\
\hline Trichilia claussenii & 4,09 & 10,94 & 13,46 \\
\hline Trichillia elegans & & & 2,50 \\
\hline Zanthoxylum rhoifolium & 4,32 & & \\
\hline
\end{tabular}

ocorreram 24 espécies, sendo que dentre estas as que apresentaram maior valor de importância 
foram: Myrocarpus frondosus (56,16), Enterolobium contortisiliquum (31,69), Ocotea puberula $(28,52)$ e Luehea divaricata $(21,29)$.

No estrato médio (Estrato 2), também ocorreram 24 espécies, sendo que o maior valor de importância foi representado por Cupania vernalis (42,28), Myrocarpus frondosus $(28,08)$, Helietta apiculata $(24,06)$ e Rollinia rugulosa $(22,19)$.

O estrato inferior (Estrato 3) apresentou 29 espécies, sendo as de maior valor de importância Cupania vernalis (41,13), Casearia sylvestris (38,39), Patagonula americana $(27,54)$ e Actinostemon concolor $(19,97)$.

A representação da ocorrência de estratos bem definidos na floresta pode ser observada na Figura 2, a qual representa a distribuição dos indivíduos na estrutura vertical, a partir dos dados coletados na parcela 10, onde evidenciou-se três estratos bem definidos na floresta.

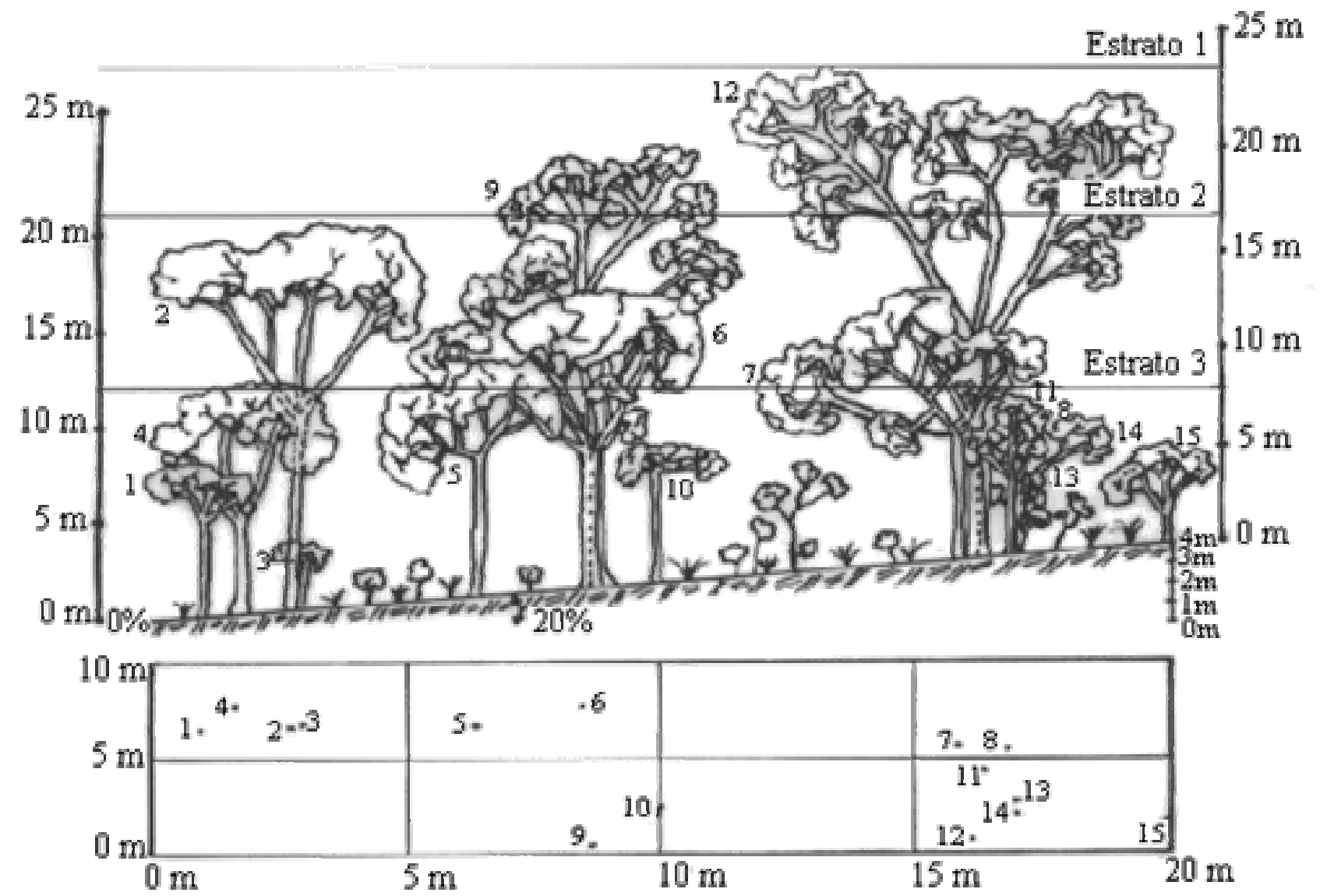

1. Myrocarpus frondosus; 2. Myrocarpus frondosus; 3. Morta; 4. Patagonula americana; 5. Sideroxylon obtusifolium; 6. Maclura tinctoria; 7. Luehea divaricata; 8. Ruprechtia laxiflora; 9. Parapiptadenia rigida; 10. Ruprechtia laxiflora; 11. Banara tomentosa; 12. Chrysophyllum marginatum; 13. Casearia sylvestris; 14. Casearia sylvestris; 15. Actinostemon concolor.

FIGURA 2: Perfil esquemático da estrutura da vegetação representando todas as árvores com CAP (circunferência a altura do peito) $\geq 30 \mathrm{~cm}$ em um fragmento florestal na Depressão Central, Santa Maria (RS).

Ciência Florestal, v. 10 , n. 2, 2000 
Relativamente à regeneração natural, foram encontradas 51 espécies vegetais (Tabela 4), sendo as de maior densidade Trichilia elegans, Nectandra megapotamica, Actinostemon concolor, Piper gaudchaudianum, Cupania vernalis, Hybanthus bigibbosus, Aiouea saligna e Parapiptadenia rigida.

Considerando as 64 espécies, que ocorreram na vegetação, dez encontram-se desde a regeneração natural até o estrato superior da floresta, ou seja, 15,62\% das espécies apresentam tendência de permanecerem na área ao longo do processo sucessional; seis espécies $(9,37 \%)$ estão presentes na regeneração e nos estratos 2 e 3; seis espécies $(9,37 \%)$ na regeneração e no estrato 3; e 22 espécies $(34,37 \%)$ somente na regeneração natural.

TABELA 4: Densidade e frequiência absoluta e relativa das espécies arbóreas e arbustivas encontradas na regeneração natural $(\mathrm{CAP}<30 \mathrm{~cm})$ amostrada em um fragmento florestal na Depressão Central, Santa Maria (RS).

\begin{tabular}{l|c|c|c|c|c}
\hline Espécie & $\mathrm{N}$ & $\mathrm{N} / \mathrm{há}$ & $\%$ Reg & Freq & Freq \% \\
\hline Acacia bonariensis & 2 & 125 & 0,311 & 6,25 & 0,578 \\
Actinostemon concolor & 88 & 5500 & 13,660 & 62,50 & 5,782 \\
Aiouea saligna & 19 & 1187,5 & 2,950 & 25,00 & 2,313 \\
Allophylus edulis & 5 & 312,5 & 0,776 & 12,50 & 1,156 \\
Allophylus guaraniticus & 6 & 375 & 0,932 & 12,50 & 1,156 \\
Cabralea canjerana & 3 & 187,5 & 0,466 & 12,50 & 1,156 \\
Calliandra tweediei & 1 & 62,5 & 0,155 & 6,25 & 0,578 \\
Casearia sylvestris & 7 & 437,5 & 1,087 & 31,25 & 2,891 \\
Cedrela fissilis & 1 & 62,5 & 0,155 & 6,25 & 0,578 \\
Celtis spinosa & 1 & 62,5 & 0,155 & 6,25 & 0,578 \\
Chrysophyllum gonocarpum & 2 & 125 & 0,311 & 12,50 & 1,156 \\
Chrysophyllum marginatum & 16 & 1000 & 2,484 & 43,75 & 4,047 \\
Cordia trichotoma & 1 & 62,5 & 0,155 & 6,25 & 0,578 \\
Cupania vernalis & 37 & 2312,5 & 5,745 & 87,50 & 8,094 \\
Daphnopsis racemosa & 1 & 62,5 & 0,155 & 6,25 & 0,578 \\
Dasyphyllum spinescens & 6 & 375 & 0,932 & 31,25 & 2,891 \\
Diospyrus inconstans & 1 & 62,5 & 0,155 & 6,25 & 0,578 \\
Enterolobium contortisiliquum & 1 & 62,5 & 0,155 & 6,25 & 0,578 \\
Eugenia involucrata & 1 & 62,5 & 0,155 & 6,25 & 0,578 \\
Eugenia uniflora & 10 & 625 & 1,553 & 31,25 & 2,891 \\
Faramea marginata & 2 & 125 & 0,311 & 12,50 & 1,156 \\
Hellietta apiculata & 10 & 625 & 1,553 & 25,00 & 2,313 \\
Hybbanthus bigibosus & 26 & 1625 & 4,037 & 43,75 & 4,047 \\
Inga marginata & 1 & 62,5 & 0,155 & 6,25 & 0,578 \\
Luehea divaricata & 10 & 625 & 1,553 & 6,25 & 0,578 \\
Machaerium stiptatum & 1 & 62,5 & 0,155 & 6,25 & 0,578 \\
Matayba elaeagnoides & 1 & 62,5 & 0,155 & 6,25 & 0,578 \\
\hline & & & & & Continua ...
\end{tabular}


TABELA 4: Continuação ...

\begin{tabular}{l|c|c|c|c|c}
\hline Espécie & $\mathrm{N}$ & $\mathrm{N} / \mathrm{ha}$ & $\%$ Reg & Freq & Freq \% \\
\hline Myrcianthes pungens & 1 & 62,5 & 0,155 & 6,25 & 0,578 \\
Myrocarpus frondosus & 3 & 187,5 & 0,466 & 18,75 & 1,735 \\
Myrsine lorentziana & 10 & 625 & 1,553 & 37,50 & 3,469 \\
Nectandra lanceolata & 8 & 500 & 1,242 & 25,00 & 2,313 \\
Nectandra megapotamica & 110 & 6875 & 17,08 & 68,75 & 6,36 \\
Ocotea puberula & 3 & 187,5 & 0,466 & 12,50 & 1,156 \\
Ocotea sp. & 1 & 62,5 & 0,155 & 6,25 & 0,578 \\
Parapiptadenia rigida & 18 & 1125 & 2,795 & 50,00 & 4,625 \\
Patagonula americana & 1 & 62,5 & 0,155 & 6,25 & 0,578 \\
Piper gaudchaudianum & 53 & 3312,5 & 8,230 & 68,75 & 6,36 \\
Rollinia rugulosa & 1 & 62,5 & 0,155 & 6,25 & 0,578 \\
Ruprechtia laxiflora & 1 & 62,5 & 0,155 & 6,25 & 0,578 \\
Sebastiania brasiliensis & 3 & 187,5 & 0,466 & 6,25 & 0,578 \\
Solanaceae & 1 & 62,5 & 0,155 & 6,25 & 0,578 \\
Solanum pseudoquina & 3 & 187,5 & 0,466 & 6,25 & 0,578 \\
Sorocea bonplandii & 6 & 375 & 0,932 & 25,00 & 2,313 \\
Styrax leprosum & 5 & 312,5 & 0,776 & 12,50 & 1,156 \\
Syagrus ronanzoffiana & 11 & 687,5 & 1,708 & 37,50 & 3,469 \\
Trichilia catigua & 3 & 187,5 & 0,466 & 12,50 & 1,156 \\
Trichilia claussenii & 22 & 1375 & 3,416 & 37,50 & 3,469 \\
Trichilia elegans & 111 & 6937,5 & 17,240 & 75,00 & 6,938 \\
Urera baccifera & 1 & 62,5 & 0,155 & 6,25 & 0,578 \\
Xylosma psedosalzmannii & 6 & 375 & 0,932 & 6,25 & 0,578 \\
Zanthoxylum naranjilo & 1 & 62,5 & 0,155 & 6,25 & 0,578 \\
TOTAL & 644 & 40250 & 100 & 1081 & 100 \\
\hline & & & & & \\
& & & & &
\end{tabular}

O grande número de espécies na regeneração constitui o banco de plântulas que persiste sob stress variável, em ambientes com baixa intensidade luminosa, sendo essas espécies tolerantes ao microclima formado pelos indivíduos dos estratos superiores. Salienta-se que muitas espécies ocorrem na regeneração, porque apresentam características de permanecerem no estrato inferior, como é o caso de Actinostemon concolor e Trichilia elegans, que pelas características próprias, não chegarão ao estrato superior da floresta. No entanto, muitas espécies dos estratos superiores não são encontradas na regeneração, por meio das suas exigências de luz para germinar, estabelecer-se e desenvolver-se. Essas espécies são conhecidas como heliófilas ou pioneiras (FINEGAN \& SABOGAL, 1988; SWAINE \& WHITMORE, 1988).

A regeneração natural apresentou maior riqueza florística do que a subpopulação adulta (indivíduos com CAP maior ou igual a $30 \mathrm{~cm}$ ), concordando com LONGHI et al. (1999) que também encontraram um grande número de espécies na regeneração, em trabalhos realizados na região. Por outro lado, o número de espécies não basta para definir um futuro de elevada riqueza para a floresta, considerando que das 22 espécies estão presentes somente na regeneração e não 
apresentam indivíduos nos demais estratos, sugerindo incerteza da permanência ou não dessas espécies na floresta.

As espécies Chorisia speciosa, Tabebuia impetiginosa e Apuleia leiocarpa ocorreram no estrato superior e não estão presentes na regeneração, podendo, em curto a médio prazo, não ocorrer em mais na floresta, fato semelhante poderá ocorrer com Machaerium stipitatum que apresenta indivíduos somente no estrato superior com baixo valor de importância e apresentou somente um indivíduo na regeneração.

\section{CONCLUSÕES}

Os resultados obtidos com a realização do presente trabalho permitiram concluir que:

1) a vegetação do fragmento estudado encontra-se em estágio de sucessão natural;

2) as famílias Lauraceae, Meliaceae, Mimosaceae, Myrtaceae e Sapindaceae foram as mais representativas da floresta;

3) Myrocarpus frondosus, Cupania vernalis, Ocotea puberula, Patagonula americana, Casearia sylvestris, Luehea divaricata, Enterolobium contortisiliquum e Helietta apiculata foram as espécies mais caraterísticas da comunidade analisada, representando $51 \%$ do valor de importância total das espécies.

\section{REFERÊNCIAS BIBLIOGRÁFICAS}

AMO RODRÍGUEZ, S. del; GÓMEZ-POMPA, A. Crecimento de estados juveniles de plantas en Selva Tropical Alta Perennifolia. In: GÓMEZ-POMPA, A.; VÁZQUEZ-YANES, C.; AMO RODRÍGUEZ, S. del et al. Regeneration de Selvas. Mexico: 1976. p. 549-565.

ARAUJO, M. M. Vegetação e banco de sementes do solo de florestas sucessionais na região do baixo rio Guamá, Benevides, Pará, Brasil. 1998. 86 p. Dissertação (Mestrado em Ciências Florestais) - Faculdade de Ciências Agrárias do Pará, Belém.

BARROS, P. L. C. de. Estudo fitossociológico de uma floresta tropical úmida no planalto Curuá-Una, Amazônia Brasileira. 1986. 146 p. Tese (Doutorado em Manejo Florestal) Universidade Federal do Paraná, Curitiba.

BEARZI, R. C.; POLTRONIERI, V. C.; LONGHI, S. J. Estrutura fitossociológica do morro das Três Marias em Santa Maria-RS. In: CONGRESSO FLORESTAL ESTADUAL, 7., 1992, Nova Prata. Anais... Santa Maria: CEPEF/FATEC/UFSM, 1992. p. 376-94.

BRASIL. Ministério da Agricultura. Divisão de Pesquisas Pedológicas. Levantamento de reconhecimento dos solos do estado do Rio Grande do Sul. Recife: 1973. 431 p.

BRAUN-BLANQUET, J. B. Fitosociologia: bases para el estudio de las comunidades vegetales. 
Madrid: H. Blume Ediciones, 1979. 829 p.

BROWER, J. E.; ZAR, J. H. Field and Laboratory Methods for General. 2. ed. Iowa: Brown Publishers, 1984. $226 \mathrm{p}$.

CAIN, S. A.; CASTRO, G. M. O. Manual of vegetation analysis. New York: Hafner Publishing Company, $1959.325 \mathrm{p}$.

DIAS, C. A.; MELlO, S. C.; CASTAMAN, D. et al. Estudo florístico e fitossociológico do município de Santa Maria, RS. I Etapa: Depressão Central - Morros Testemunha. In: SIMPÓSIO SOBRE ECOSSISTEMAS NATURAIS DO MERCOSUL, 1., Santa Maria, 1996. Anais ... Santa Maria: UFSM/CEPEF, 1986. p. 97-118, 1996.

FINEGAN, B.; SABOGAL, C. El desarrolo de sistemas de produccion sostenible en bosque tropical Humedos da Bajura: un estudio de caso en Costa Rica ( $1^{a}$ parte). El Chasqui, v. 17, p. 3-21, 1988.

FINOL, H. Nuevos parametros a considerarse en el análisis estructural de las selvas virgenes tropicales. Revista Forestal Venezolana, v. 21, p. 29-42, 1971.

FINOL, H. La silvicultura en la Orinoguia Venezolana. Revista Forestal Venezolana, v. 25, p. 37112, 1975.

GÓMEZ-POMPA, A.; WIECHERS, B. L. Regeneration de los ecosistemas tropicales y subtropicales. In: GÓMEZ-POMPA, A.; VÁZQUEZ-YANES, C.; AMO RODRÍGUEZ, S. del et al. Regeneration de selvas. Mexico: Continental, 1976. p.11-30.

HERRMANN, M. L. de P.; ROSA, R. de. O Relevo. In: IBGE. Geografia do Brasil: região sul. Rio de Janeiro, 1990. p. 55-84.

JARDIM, F. C. S.; HOSOKAWA, R. T. Estrutura da floresta equatorial úmida da Estação Experimental de Silvicultura Tropical do INPA. Acta Amazonica, v. 16/17, p. 411-508, 1986.

KAUL, P. F. T. Geologia. In: IBGE. Geografia do Brasil: região sul. Rio de Janeiro, 1990. p. 2954.

LAMPRECHT, H. Ensaio sobre la estructura floristica de la parte Sur-oriental del Bosque Universitario "El camital"- Estado Burina. Revista Forestal Venezolana, v. 7, n. 10-11, p. 1763, 1964.

LONGHI, S. J. A estrutura de uma floresta natural de Araucaria angustifolia (Berth.) Kuntze, no sul do Brasil. 1980. 198 p. Dissertação (Mestrado em Ciências Florestais) - Universidade Federal do Paraná, Curitiba.

LONGHI, S. J. Agrupamento e análise fitossociológica de comunidades flroestais na sub-bacia hidrográfica do Rio Passo Fundo - RS. 1997. 198 p. Tese (Doutorado em Ciências Florestais) - Universidade Federal do Paraná, Curitiba.

LONGHI, S. J.; NASCIMENTO, A. R. T.; FLEIG, F. D.; DELLA-FLORA, J. B.; FREITAS, R. A. de; CHARÃO, L. W. Composição florística e estrutura da comunidade arbórea de um fragmento florestal no município de Santa Maria-Brasil. Ciência Florestal, v. 9, n. 1, p. 115-33, 1999.

Ciência Florestal, v. 10 , n. 2, 2000 
MACHADO, P. F. dos; LONGHI, S. J. Aspectos florísticos e fitossociológicos do "morro do Elefante", Santa Maria, RS. Rev. Centro de Ciências Rurais, v. 20, n. 3-4, p. 261-80, 1992.

MARTINS, F. R. Estrutura de uma Floresta Mesófila. Campinas: UNICAMP, 1991. 246 p.

MORENO, J. A. Clima do Rio Grande do Sul. Porto Alegre: Secretaria da Agricultura, 1961. 42 p.

PIELOU, E. C. Mathematical Ecology. New York: John Wiley \& Sons, 1977. 385 p.

SARTORI, M. da G. B. O clima de Santa Maria, RS: do regional ao urbano. 1979. 165 p. Dissertação (Mestrado em Geografia) - Departamento de Geografia, Universidade de São Paulo, São Paulo.

SILVA, L. C. da; LONGHI, S. J. Aspectos florísticos do morro do Link, Santa Maria, RS. In: SEMANA DO MEIO AMBIENTE, 3., 1989, Piracicaba. Anais... Piracicaba: USP/CENA, 1989. p. 177-207.

SWAINE, M. D.; WHITMORE, T. C. On definition of ecological species groups in tropical rain forest. Vegetatio, n. 75, p. 81-86, 1988.

VIEIRA, I. C. G. Forest succession after shifting cutivation in Eastern Amazonia. 1996. 205 p. These (Doutorado of Philosophy) - University of Stirling, Scotland. 